\title{
Discours
}

Revue de linguistique, psycholinguistique et

informatique. A journal of linguistics, psycholinguistics

and computational linguistics

$2 \mid 2008$

Varia

\section{Réduction et intégration de prédications : paramètres pour l'analyse des co-prédications adjectivantes}

Reduction and integration of predications: parameters for the analysis of adjectival co-predications

\section{Eva Havu et Michel Pierrard}

\section{(2) OpenEdition}

Journals

Édition électronique

URL : http://journals.openedition.org/discours/1042

DOI : $10.4000 /$ discours. 1042

ISSN : 1963-1723

Éditeur :

Laboratoire LATTICE, Presses universitaires de Caen

\section{Référence électronique}

Eva Havu et Michel Pierrard, « Réduction et intégration de prédications : paramètres pour l'analyse des co-prédications adjectivantes », Discours [En ligne], 2 | 2008, mis en ligne le 02 juillet 2008, consulté le 01 mai 2019. URL : http://journals.openedition.org/discours/1042 ; DOI : 10.4000/discours. 1042

Ce document a été généré automatiquement le 1 mai 2019.

\section{(c) $($ ) $\odot \odot$}

Discours est mis à disposition selon les termes de la licence Creative Commons Attribution - Pas d'Utilisation Commerciale - Pas de Modification 4.0 International. 


\title{
Réduction et intégration de prédications : paramètres pour l'analyse des co-prédications adjectivantes
}

\author{
Reduction and integration of predications: parameters for the analysis of \\ adjectival co-predications
}

Eva Havu et Michel Pierrard

\section{Cadre d'analyse}

1 La 'prédication seconde' est globalement définie au plan sémantico-syntaxique comme "un type de séquence qui, malgré son statut syntaxiquement intégré, exprime sémantiquement un contenu phrastique à l'intérieur même de la phrase » (Cadiot, Furukawa, $2000: 3)$. Cette définition assez extensive recouvre le fonctionnement d'une gamme de constructions à première vue assez disparates: épithètes détachées et appositions [1a-b], attributs de l'objet directs et indirects [1c-d], constructions absolues, infinitives, relatives [1e-g], etc. :

\footnotetext{
[1a] Furieux, le client est parti.

[1b] L'hirondelle, messagère du printemps, a fait son apparition dans notre région.

[1c] Luc a connu Pierre enfant.

[1d] Luc traite Paul d'enfant.

[1e] Il est rentré chez lui les mains dans les poches.

[1f] Je les entends chanter.

[1g] Les femmes, qui étaient malades, ont été abandonnées.
}

Elle peut également amener à pointer des traits définitoires multiples [pour un aperçu des traits, (Havu, Pierrard, 2004)]. Comment concilier cette variété de structures et de 
traitements avec la cohérence d'une fonction sémantico-syntaxique unique ? Dans la présente contribution, nous nous restreindrons aux tours que nous désignerons par le terme de co-prédications adjectivantes ${ }^{1}$ (coP-Adj), soit les constructions de type [X [ $\left.\mathrm{SN}_{1}\right]$ ${ }_{\text {Préd1 } 1} \mathrm{Adj}_{\mathrm{Préd} 2}$ où le thème $\mathrm{SN}_{1}$ de la prédication secondaire remplit une fonction dans la prédication centrale (préd1) et où le co-prédicat a la forme d'un adjectif ou d'un participe. Les travaux sur la question (Combettes, 1998 ; Lagae, 1998 ; Riegel et al., 1998; Cadiot, Furukawa, 2000 ; Forsgren, 2000 ; etc.) y répertorient généralement les tours :

[1a] Furieux, le client est parti.

[2a] Je bois le café chaud.

[2b] Paul trouve la mesure indispensable.

[2c] Luc rend Marie heureuse.

[3a] Il a qualifié l'équipe d'excellente.

[3b] Il considère cette affaire comme importante.

[4] Il a les dents longues.

[5] Il y a une place de libre.

3 Notre étude prendra donc en considération cinq types de coP-Adj soient T1 : les adjoints [1] ; T2 : les attributs directs de l'objet avec des verbes occasionnellement attributifs [2a], des verbes à attributs propositionnels [2b] et des verbes causatifs d'état [2c]; T3: les attributs indirects de l'objet, introduits par une préposition [3a] ou par comme [3b] ; T4 : les emplois attributifs avec avoir [4] ; T5 : les tours en de adj [5].

4 L'objectif de cette contribution est de proposer un cadre permettant d'appréhender la multiplicité des paramètres qui gouvernent les importantes différences d'extension et de flexibilité de ces tours regroupés sous l'étiquette des coP-Adj. Notre approche suggère plus particulièrement l'application aux constructions de type $\left[\mathrm{X}\left[\mathrm{SN}_{1}\right]_{\text {Préd1 }} \mathrm{Adj}\right]_{\text {Préd2 }}$ d'une série de propriétés avancées pour décrire les différents degrés de connexion entre propositions. L'hypothèse d'un tel traitement parallèle est pleinement compatible avec la définition de départ de la prédication seconde qui la voit comme le produit d'une connexion de prédicats (Cadiot, Furukawa, 2000). Plusieurs types de paramètres sémantico-syntaxiques ont été développés pour hiérarchiser les divers types de connexion et de dépendance phrastiques et sont applicables aux coP-Adj. Nous optons dans cette étude pour deux des trois dimensions du traitement paramétrique des types de connexions phrastiques, proposées par Lehmann (1989) et généralement reconnues comme étant une des approches analytiques les plus appropriées des combinaisons de propositions dans les langues naturelles (Raible, 2001: 614):

- Une première dimension oppose les traits [EXPANSION] et [RÉDUCTION] : « the degree to which the subordinate clause is expanded or reduced » (Lehmann, $1988: 193$ ). Elle permet de caractériser le degré d'expansion/ de réduction des propriétés propositionnelles de la subordonnée. Ainsi, par exemple, une infinitive sera analysée comme plus réduite qu'une complétive ;

- La deuxième dimension distingue les traits [AUTONOMIE] et [INTÉGRATION] : « The requirement in our definition that a subordinate construction must be part of a higher construction leaves much room for variation » (Ibid. : 183). Celle-ci permet d'inférer une échelle scalaire visant à spécifier le niveau d'intégration de la subordonnée dans la régissante. De ce point de vue, une corrélative sera moins intégrée qu'une complétive.

5 Ces deux dimensions permettent de définir des paramètres de hiérarchisation qui seront appliqués aux divers types de coP-Adj et qui devraient favoriser un traitement convergent de leur diversité de fonctionnement : 
La dimension [EXPANSION] vs [RÉDUCTION] vise en premier lieu la cohésion intraprédicative de coP-Adj : en quelle mesure la co-prédication est-elle perçue comme une entité distincte ? Et le rapport associatif entre les composantes de la co-prédication est-il souligné ou au contraire escamoté ? Enfin, est-ce que coP-Adj restreint l'apport sémantique de préd1 ou, au contraire, n'a pas d'impact sur la saisie de la prédication centrale?

7 La dimension [AUTONOMIE] vs [INTÉGRATION] engage pour sa part plutôt la cohésion interprédicative entre préd1 et coP-Adj. L'examen d'un certain nombre de marques fonctionnelles et rectionnelles permettra de différencier les co-prédications adjectivantes selon le degré de hiérarchisation du rapport interprédicatif et le niveau d'intégration du co-prédicat dans le prédicat recteur.

8 Dans les points suivants du texte, nous évaluerons la pertinence du cadre proposé pour l'appréhension de l'ensemble des tours regroupés sous l'étiquette de coP-Adj.

\section{Cohésion intraprédicative : le trait [+/-EXPANSION]}

9 Le rapport co-prédicatif entre $\mathrm{SN}_{1}$ et Adj est-il rendu saillant ou, au contraire, la cohésion entre ces deux composantes est-elle plutôt occultée ? L'évaluation du degré de cohésion intraprédicative du co-prédicat adjectivant [+/- EXPANSION] prendra pour points de référence les traits basiques suivants d'un rapport prédicatif :

- la transparence du lien thème-prédicat ;

- la saisie de la co-prédication comme argument global ;

- le degré de complétude du prédicat.

\subsection{La transparence du lien thème - prédicat}

Certaines marques morphologiques ou fonctions syntaxiques obscurcissent le lien associant $\mathrm{SN}_{1}$ au co-prédicat adjectivant et entravent ainsi la perception de la cohésion intraprédicative. L'occultation du lien associatif peut jouer sur l'identification de $\mathrm{SN}_{1}$, thème de la coP-Adj; elle peut aussi porter sur le rapport entre $\mathrm{SN}_{1}$ et le prédicat adjectivant.

11 (a) L'absence de contraintes fonctionnelles pesant sur $\mathrm{SN}_{1}$ atténue fortement sa perception comme thème de coP. Ainsi, dans T1, il peut remplir des fonctions diverses dans la phrase, y compris une fonction indirecte, éventuellement hiérarchiquement subordonnée à un autre $\mathrm{SN}$ dans la structure argumentale de préd1 :

[6a] La mère de la petite Sophia, trouvée morte mardi dernier [...], a déclaré [...]. (Le Figaro 01.03.03: 9).

12 (b) Dans d'autres tours [6b-e : T2a, T2c, T3a-b], le SN est fonctionnellement plus contraint mais peut cependant, par transformation passive, remplir la fonction sujet dans préd1, ce qui entraîne nécessairement une séparation entre le SN et l'adjectif prédicatif :

[6b] Il a acheté cette robe neuve / Cette robe a été achetée neuve.

[6c] Luc a rendu Marie heureuse / Marie a été rendue heureuse par Luc.

[6d] Il qualifie cette nouvelle de prématurée / Cette nouvelle est qualifiée de prématurée.

[6e] Il considère cette affaire comme importante / Cette affaire est considérée comme importante. 
Pour T2c, la rupture fréquente de l'accord N-Adj à l'écrit, lorsque ce dernier est antéposé, accentue l'apport sémantique crucial du prédicat second auprès du verbe recteur au détriment de la cohésion du co-prédicat. $\mathrm{SN}_{1}$ devient en quelque sorte $\mathrm{OD}$ d'un prédicat complexe, composé du verbe recteur et de l'adjectif prédicatif :

[7a] [...] en gardant présent à l'esprit [...] la mise en fonctionnement du langage. (Allain-Sokolsky; Grevisse-Goosse, 1991, § $297: 492$ )

[7b] [...] un irréalisme qui rend plus que hasardeux toute tentative [...] de rapprochement. (Noblecourt; Ibid.)

[7c] Il a fallu rendre payant cette action. (Le Soir $06.10 .06: 10$ )

(c) Par contre, dans quelques types, $\mathrm{SN}_{1}$ tend à être [6f-f': T2b] ou est [6g-h : T4, T5] fonctionnellement contraint en position d'objet, ce qui renforce la cohésion de coP :

[6f] Jean croit Luc malade / Jean trouve Luc impoli.

[6f] * Luc est cru malade / ?Luc est trouvé impoli par Jean / Luc a été trouvé très impoli.

[6g] Il a les dents longues / *Les dents sont eues longues (par lui).

[6h] Pierre a dix cravates de rouges (Wilmet, 2003:568)/* Dix cravates de rouges se trouvent dans la vitrine.

De plus, l'entité que constituent le $\mathrm{SN}_{1}$ et l'adjectif prédicatif dans le cas du type $\mathrm{T} 2 \mathrm{~b}$ est soulignée par l'absence fréquente d'accord du participe passé du verbe régissant avec l'OD antéposé. Selon le Bon usage, il s'agit d'une indication manifeste de la cohésion du nom thème et de l'adjectif, ressentis comme un ensemble :

[8a] Une vie qu'on aurait voulu belle. (Maurois ; Grevisse-Goosse, 1991, § 914 :

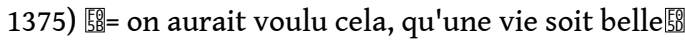

[8b] J'assistais à des événements que mon grand-père eût certainement jugé invraisemblables. (Sartre; Ibid.)

国= mon grand-père aurait jugé ceci, que ces événements étaient

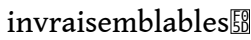

(d) Dans trois cas |T3a, T3b, T5 : 6d,e,h], le rapport - indirect - entre $\mathrm{SN}_{1}$ et le co-prédicat instille l'impression d'un double lien rectionnel (direct et indirect) avec le verbe de préd1 (Il la qualifie de prématurée Il la reçoit de sa mère) ou d'un rapport hiérarchisé au sein d'un SN (une place de libre la place de mon frère) et obscurcit dès lors la cohésion de la coprédication. Notons que dans le cas de considérer (comme), le caractère indirect du lien entre $\mathrm{SN}_{1}$ et l'adjectif est sujet à une certaine variabilité :

[9] Les étudiants, qui se sont beaucoup émus de ce projet, considéreront-ils le geste suffisant? (Le Monde 04.03.94: 17)

Bref, la cohésion entre les composantes est rendue plus opaque dans T1, marqué par conséquent du trait [- EXPANSION], face aux tours $\mathrm{T} 2 \mathrm{~b}$ et $\mathrm{T} 4$, qui accentuent nettement la cohésion intraprédicative [+ EXPANSION]. T3a et $\mathrm{b}$ ainsi que T5 tendent plutôt vers le pôle [-EXPANSION], tandis que T2a et T2c, pour leur part, tendent plutôt vers [+ EXPANSION].

\subsection{La saisie de la co-prédication comme argument global}

18 La caractéristique de base des co-prédications adjectivantes est que le thème $\mathrm{SN}_{1}$ de coP remplit une fonction dans préd1 sans impliquer Adj [- EXPANSION]. Une marque de 
saillance du lien co-prédicatif sera alors le fait que coP dans sa totalité remplit une fonction argumentale dans préd1 ([+ EXPANSION]). Dans le premier cas de figure ([EXPANSION]), l'énoncé avec coP-Adj est parfaitement compatible avec celui sans prédicat second; dans le second cas par contre [+ EXPANSION], il y a effectivement incompatibilité entre les deux constructions :

[10a] Le client est parti, furieux $\sim$ Le client est parti.

$[10 \mathrm{~b}]$ Je bois le café chaud $\sim$ Je bois le café.

[10c] Paul trouve la mesure indispensable $\neq$ Paul trouve la mesure.

[10d] Luc rend Marie heureuse $\neq$ Luc rend Marie.

[10e] Il a qualifié l'équipe d'excellente $\neq$ Il a qualifié l'équipe.

[10f] Il considère cette affaire comme importante $\neq \mathrm{Il}$ considère cette affaire.

$[10 \mathrm{~g}] \mathrm{Il}$ a les dents longues $\neq \mathrm{Il}$ a des dents.

[10h] Il y a une place de libre $\sim$ Il y a une place.

Le fonctionnement des types $\mathrm{T} 1, \mathrm{~T} 2 \mathrm{a}$ et $\mathrm{T} 5[10 \mathrm{a}, \mathrm{b}, \mathrm{h}]$ indique donc que la coP n'est pas appréhendée comme un argument global de préd1 ; par contre, dans T2b-c, T3 et T4 [10cg], c'est bien coP dans son ensemble qui intervient en tant qu'argument du verbe principal. L'analyse est confirmée par les restrictions sémantiques imposées à l'objet $\mathrm{SN}_{1}$ [11a-b] : lorsque coP-Adj dans sa totalité remplit la fonction d'argument auprès de préd1 [11a], les restrictions sont imposées uniquement par le co-prédicat et pas par le prédicat régissant. Par contre, lorsque $\mathrm{SN}_{1}$ remplit seul cette fonction [11b], le co-prédicat adjectivant n'interfère pas au niveau des contraintes (cf. aussi Guimier, 1998) :

[11a] *Je croyais la route/ la route est praticable/ Je croyais la route praticable.

[11b] *Je bois la viande/ la viande est tendre/ *Je bois la viande tendre.

\subsection{La perception du co-prédicat comme nœud verbal}

La caractéristique générale des co-prédicats adjectivants est qu'ils ne contiennent pas de forme verbale finie, contrairement aux structures attributives classiques. Cela n'implique toutefois pas l'absence totale de forme verbale. Certains tours autorisent en particulier l'emploi de participes présents, ce qui accentue évidemment la saillance du rapport coprédicatif ([+ EXPANSION]) :

[1a'] Refusant de payer, le client est parti.

[2a'] J'ai trouvé Marie dormant sur le canapé.

$\left[2 b^{\prime}\right]$ *Il croit Pierre cherchant du boulot.

$\left[2 c^{\prime}\right]$ * Luc rend Marie trouvant du boulot.

[3a'] *Il a qualifié l'équipe de manquant d'ambition.

[36'] Il considère cette affaire comme méritant la plus haute attention.

$\left[4^{\prime}\right]$ *Il a les dents étant longues.

[5'] *Il y a une place d'étant libre.

21 Les tours T1, T2a et T3b permettent l'emploi de participes présents, contrairement aux autres tours. Certains emplois $[12 \mathrm{a} ; 12 \mathrm{c}]$ pourraient laisser supposer que T2b permet également l'expansion par le participe présent :

[12a] Il a vu Pierre embrassant Jeanne.

[12b] *Il a constaté Pierre embrassant Jeanne.

[12c] Il croit Pierre affirmant qu'il cherche du boulot. 
[12d] *Il pense Pierre affirmant qu'il cherche du boulot. pour déterminer si le caractère co-prédicatif des séquences $S N_{1}+$ Adj est saillant [+ EXPANSION] ou si au contraire la cohésion intraprédicative dans coP est plutôt réduite, ce

[13a] Amenant sa femme à la mer, il eut un accident/ L'y amenant, il eut un accident/ le lui disant, je n'étais pas fier.

[13a'] *Y malade, il est revenu sans attendre la fin des vacances.

[13b] Devant/ pouvant relire intégralement son manuscrit, il corrigea page par page les fautes.

(b) l'apport de la structure argumentale d'un prédicat verbal :

Dans le cas d'un participe présent co-prédicatif, les expansions du lexème verbal correspondent aux divers compléments que le verbe accepte [13c-d] :

[13c] Lui ayant déjà offert un cadeau au Mexique, il ne veut plus lui en offrir un à Cuba.

[13d] Estimant la chose impossible, il y renonça.

(c) l'apport de la valeur sémantique associée au lexème et à sa flexion

Les rapports sémantiques exprimés par le participe présent co-prédicatif sont infiniment plus variés que la 'relation en être' qu'on trouve avec l'adjectif [13e-f]. Par ailleurs, sous la flexion du participe présent, le verbe «envisage le procès en cours de déroulement (aspect inaccompli)» [13g-h] ; (Riegel et al., 1999 : 341) :

[13e] Content, il est parti > Il était content et...

[13f] Quittant sa mère, il prononça ces mots/ ?? Il était quittant et...

[13g] Il a vu Pierre s'asseoir/ s'asseyant/ assis/ heureux à côté de Jeanne.

[13h] Il a vu Pierre embrasser/ embrassant/ *embrassé Jeanne (embrassé par Jeanne).

Les tours T2b, T2c, T3a, T4 et T5 ne permettent pas un renforcement de la perception de l'entité co-prédicative par l'emploi d'un participe présent [- EXPANSION] ; les types T1, T2a et T3b, par contre, peuvent renforcer de cette manière la saillance du coP [+ EXPANSION].

\subsection{Conclusions} La transparence ou au contraire l'opacité du lien entre le thème et le co-prédicat ainsi 
qui induit un rapport prédicatif opacifié [- EXPANSION]. Toutefois, l'emploi d'une forme du participe présent permet de renforcer la perception du co-prédicat comme nœud verbal dans les tours où la cohésion intraprédicative est faible (T1, T2a) :

\begin{tabular}{|l|c|c|c|c|c|c|c|c|}
\hline & T1 & T2a & T2b & T2c & T3a & T3b & T4 & T5 \\
\hline Lien SN 1 -Adj & - & $+/-$ & + & $+/-$ & $-/+$ & $-/+$ & + & $-/+$ \\
\hline Argument global & - & - & + & + & + & + & + & - \\
\hline Co-prédicat comme nœud verbal & + & + & - & - & - & + & - & - \\
\hline
\end{tabular}

Tableau 1 : cohésion intraprédicative

La cohésion de la co-prédication, soit le trait [+ EXPANSION], est incontestablement la plus marquée dans les tours à attributs propositionnels [T2b] et les emplois attributifs avec avoir [T4] ; ce marquage est aussi présent, mais dans une moindre mesure, avec des verbes causatifs d'état [T2c]. L'appréhension est plus contradictoire avec les attributs indirects en comme [T3b] mais surtout avec ceux introduits par une préposition [T3a]. Ces tours combinent des indices qui mettent en exergue le rapport cohésif avec un lien indirect entre composantes, ce qui marque par contre une déstructuration de l'entité. Dans le cas des verbes occasionnellement attributifs [T2a], mais surtout dans les tours en de + adj [T5] et les adjoints [T1], la cohésion est la moins évidente [- EXPANSION] : coP n'est pas un argument global du verbe régissant et le lien $\mathrm{N}_{1}$-Adj est occulté, dans T5 par la structure indirecte de la construction. Certaines constructions, qui contiennent plutôt le trait [EXPANSION] - surtout $\mathrm{T} 1$ et dans une moindre mesure T2a et T3b - peuvent cependant souligner de manière interne la cohérence de la co-prédication en sélectionnant en tant que co-prédicat un participe présent qui renforce la perception de celui-ci comme nœud verbal.

\section{Cohésion interprédicative préd1-préd2 : [+/- INTÉGRATION]}

Le degré croissant d'intégration de la co-prédication dans la prédication centrale ou au contraire le maintien d'une certaine autonomie entre les prédications [+/- INTÉGRATION] se traduira par un certain nombre d'indices fonctionnels et rectionnels. Nous examinerons plus précisément :

- Le degré d'imbrication des constructions (dépendance fonctionnelle);

- le niveau d'intégration de coP par rapport au prédicat régissant (dépendance rectionnelle).

Ces deux dimensions fourniront des indications sur le degré de cohésion interprédicative.

\subsection{Dépendance fonctionnelle}

Deux propriétés fonctionnelles doivent permettre d'évaluer le degré d'interdépendance des prédications: l'incorporation ou non de la co-prédication dans la portée de la 
négation de la prédication centrale et le type de rapport de hiérarchisation qui s'instaure entre les prédications. Le positionnement des différents tours par rapport à ces propriétés indique le degré de flexibilité fonctionnelle des coP et déterminera leur caractère +/- intégré.

\subsubsection{Portée de la négation}

[14a] Luc ne boit pas son thé chaud.

[interprétation : Il le boit non chaud]

[14b] Il ne considère pas cette mesure comme indispensable.

[interprétation : Il la considère comme non indispensable]

[14c] Il n'a pas les yeux bleus.

[interprétation : Il les a non bleus]

[14d] Jeune, il ne faisait pas de sport.

[interprétation : *Non jeune, il faisait du sport]

[14e] Il n'a pas trouvé de place de libre.

[interprétation : *Il a trouvé des places non libres (Lagae 1998 : 59)]

Dans le cas de T1 (Combettes, $1998:$ 13) et de T5 (Lagae, 1998 : 59) en effet, la portée de la négation n'affecte que la prédication première et n'a pas d'impact sur coP, comme l'illustre l'impossibilité des interprétations dans [14d-e].

Le fait que la co-prédication puisse être niée de manière autonome sera pour sa part un indice de non-attraction de coP dans la structure argumentale de préd1 :

[15a] Le client est parti, mais pas furieux.

[15b] Paul a acheté sa voiture mais pas neuve/ Luc boit son thé mais pas

chaud.

[15c] *Paul juge la mesure mais pas indispensable.

$[15 \mathrm{~d}]{ }^{*}$ Cette nouvelle a rendu la mesure mais pas indispensable.

[15e] *Il tient cette nouvelle mais pas pour vraie.

[15f] *Il considère cette mesure mais pas comme indispensable

$[15 \mathrm{~g}]$ *Il a les yeux mais pas bleus.

[15h] *Il a trouvé une place mais pas de libre.

Dans le cas de T1 et de T2a donc [15a-b], la possibilité d'accepter une polarité négative spécifique souligne la non-intégration de coP dans la structure argumentale de préd1 [+ AUTONOMIE]. Cette polarité négative spécifique n'existe plus dans les autres types.

41 En résumé, les deux tests sur la négation soulignent tout particulièrement une autonomie de coP vis-à-vis de la structure argumentale de préd1 dans les types T1, T2a et T5, plutôt orientés donc vers le pôle [- INTÉGRATION]. 


\subsubsection{Niveau de hiérarchisation des prédications}

42 Le type de subordonnée permettant de paraphraser coP-Adj donne une indication sur le niveau de hiérarchisation et donc sur le degré plus ou moins grand d'autonomie ou d'intégration fonctionnelle des structures co-prédicatives : si l'énoncé paraphrastique est une circonstancielle ou une relative explicative, ce sera l'indication d'une autonomie consistante par rapport à la structure argumentale du prédicat enchâssant ou à l'un des arguments de celui-ci [16a-b : T1 en T2a]; si par contre l'énoncé paraphrastique prend la forme d'une que-phrase ou d'une relative prédicative, il faudra l'interpréter comme le signe d'une autonomie plus limitée et d'une intégration plus forte dans la structure argumentale du prédicat ou dans un des arguments de celui-ci [16c,f-h: T2b, T3b, T4 en T5]; l'impossibilité de paraphrase directe sera enfin l'expression d'une intégration très poussée [16d-e : T2c en T3a] :

[16a] Furieux, le client est parti - \{Le client, qui était furieux, ... / Comme il était furieux, le client ...\}

[16b] Paul a acheté sa voiture neuve - $\{. .$. la voiture, qui était neuve / ... alors qu'elle était neuve\}

[16c] Paul juge la mesure indispensable - \{Paul juge que la mesure est indispensable\}

[16d] Cette nouvelle rend la mesure indispensable - \{pas de paraphrase immédiate\}

[16e] Il tient la mesure pour indispensable - \{pas de paraphrase immédiate\}

[16f] Il considère la mesure comme indispensable - \{ll considère que la mesure est indispensable\}

[16g] Il a les yeux bleus - \{Il a les yeux qui sont bleus\}

[16h] Il y a une place de libre - \{ll y a une place qui est libre\}

Outre le type d'énoncé paraphrastique, relevons aussi la variété décroissante des paraphrases possibles; ainsi, pour T1, Havu (2002) a relevé de nombreux effets de sens pour les paraphrases circonstancielles:

[17a] La recette, simple dans son principe, exigeait complexité et subtilité.

[...] Habité par une émotion.... (Le Monde 14.11.01 :33)

(= bien qu'elle soit simple dans son principe / la recette, qui était simple dans son principe)

[17b] Conscient de la faiblesse financière et politique de l'état postcommuniste, le président Boris Eltsine ne se pressa jamais d'ouvrir les hostilités contre les barons régionaux. (Le Figaro $15.5 .00: 4$ )

(= comme il était conscient / le président, qui était conscient)

[17c] [...] Il faut cependant attendre plusieurs siècles avant de voir renaître la statuaire proprement dite. Rare et maladroite à l'époque romane, à l'exception du portail de l'église d'Andlau, elle s'épanouit à l'ère des grandes constructions gothiques. (Guide Alsace 98)

(= alors qu'elle était rare et maladroite / après avoir été rare et maladroite)

De même, en ce qui concerne T2a, Muller (2000: 24-30) remarque qu'il faut inclure dans l'interprétation du rapport entre le verbe et l'attribut second un certain degré de variabilité d'après le contexte :

[17d] Il boit son café tiède. (= Il boit son café quand le café est tiède)

[17e] Ses petits amis, elle les choisit riches. (= ...s'ils sont riches)

[17f] Ses petits amis, elle les a (toujours) choisis riches. (= ...parce que riches) 
[18a] Content, Jean a parlé à Marie.

[18b] On apercevait, immobile, un homme qui [...] (Combettes, 1998 : 77).

[18c] Le petit garçon était blotti dans les bras de son père, muet de frayeur (deux interprétations).

[18d] La mère de la petite Sophia, trouvée morte mardi dernier [...], a déclaré

[...] (Le Figaro 01.3.03 : 9)

Par ailleurs, les coP-Adj peuvent avoir non seulement un pronom clitique sujet, OD ou OI comme thème mais encore le référent d'un adjectif possessif [19c] par.exemple (Berrendonner, Reichler- Béguelin, 1989:119) :

[19a] Jeune, je faisais du sport.

[19a] Allongée sur le divan bas, la lampe voilée la laissait presque tout entière dans l'ombre (Combettes 1998 : 38).

[19c] Jeune, mon idole était Jouvet (Le Figaro $15.05 .00: 36$ ).

\section{Apport argumental variable de la co-prédication :}

51 Le point de connexion est nécessairement un argument central du verbe de préd1, sujet ou objet (Je bois mon café chaud/ *Je mets du sucre dans mon café chaud). Dans les types T2a,c et T3a,b, l'énoncé peut être mis au passif, et le COD devient alors le sujet de la phrase :

[20a] Il a acheté cette robe neuve / Cette robe a été achetée neuve.

[20b] Luc a rendu Marie heureuse / Marie a été rendue heureuse par Luc.

[20c] Il qualifie cette nouvelle de prématurée / Cette nouvelle est qualifiée de prématurée.

[20d] Il considère cette affaire comme importante / Cette affaire est considérée comme importante. 


\section{Apport argumental contraint de la co-prédication :}

[21a] Paul trouve/ estime la mesure indispensable/ Paul trouve Jean impoli.

[21a'] ?La mesure est trouvée/ estimée indispensable/ * Jean est trouvé impoli.

[21a"] La mesure a été trouvée/ estimée indispensable/ Jean a été trouvé très impoli.

[21b] Jean croit Luc malade.

[21b'] * Luc est cru malade.

[21b"] ?? Luc a été cru malade.

3 Si l'acceptabilité des énoncés T2b peut varier selon le verbe ou le temps [comparez [21a'] vs [21a"] avec [21b'] vs [21b"]], l'apport argumental est totalement contraint dans les énoncés de type $\mathrm{T} 4$ et $\mathrm{T} 5$ :

[22a] Il a les yeux bleus / Jean a la répartie facile.

[22b] * Les yeux sont eus bleus (par lui) / *La répartie est eue facile (par Jean).

[22c]? Les yeux bleus sont beaux/ ?La répartie facile est la spécialité de Pierre.

Dans la construction du type 4 , le thème de coP sera toujours intégré dans préd1 en fonction OD, car la construction ne peut pas subir de transformation passive. Si l'ensemble du segment co-prédicatif passe en position de sujet [22c], son interprétation passera nécessairement par une lecture épithète, la lecture co-prédicative étant bloquée. Dans T5 aussi, la coP-Adj est liée à la position objet de son thème auprès du préd1 dont elle dépend [22d-e]. Fréquemment, il s'agit en réalité d'une fonction de complément des présentatifs il y a ou voici, comme dans [22f-g] (Noailly, 1999 : 85) :

[22d] Pierre a dix cravates de rouges / de souillées.

[22e] * Dix cravates de rouges/ de souillées se trouvent dans la vitrine.

[22f] Il y a [...] un détail de bizarre.

$[22 \mathrm{~g}]$ * Un détail de bizarre m'a été raconté.

5 En résumé, sur base de la flexibilité du point de connexion de la co-prédication, nous avons distingué sur l'axe allant de [- INTÉGRATION] vers [+ INTÉGRATION] une gradation partant d'un apport variable [T1], argumental [T2a, c et T3a,b], et enfin argumental contraint $[\mathrm{T} 2 \mathrm{~b}$ et surtout $\mathrm{T} 4$ et $\mathrm{T} 5\}$.

\subsubsection{Apport sémantique de la co-prédication}

56 L'hypothèse d'un lien entre l'apport de la co-prédication à la rection du verbe central et l'accroissement de son poids sémantique dans le prédicat principal parait pertinente.

Dans le cas de T1 et de T2a, préd1 admettra une large gamme de verbes qui conservent leur sens plein et coP ne constitue qu'un apport accessoire. Dès T2b ou T3a-b cependant, on constate un poids croissant du co-prédicat dans la prédication centrale et en conséquence une désémantisation du verbe régissant : verbes 'faibles' d'opinion, exigeant la présence d'un élément compensatoire, dans T2c, des causatifs d'état (Pierrard, 2002 : 
366). Dans ces cas-là, le co-prédicat adjectival fait corps en réalité avec un verbe qui a perdu une partie de son indépendance cf. (Blanche-Benveniste, Caddéo, 2000 : 62-63 ; Muller, 2000 : 33 ; Pierrard, 2002 : 365-367). Dans T2c, on peut même estimer que le verbe forme avec le co-prédicat adjectivant une unité inséparable, souvent remplaçable par une forme verbale synthétique (Pierrard, 2002:363: La vue des crocodiles rend Nicole triste > attriste Nicole).

Le poids sémantique de la co-prédication est particulièrement saillant dans T4 et T5 :

[23a] Jean a la répartie facile.

[23b] Il y a/ voici/ il reste/ tu avais une place de libre.

Dans [23b], préd1 se limite en fait à être un " actualisateur » de coP, puisque la grande majorité des introducteurs relevés sont des formes comme il y a, c'est, il reste, voilà (Lagae, 1998); pour [23a], les études sur la question soulignent que les contraintes formelles pesant sur le $\mathrm{N}_{1}$ rendent inutile l'expression d'un sens de possession par le verbe avoir. Celui-ci devient alors une sorte d' " adhésif » qui colle le $\mathrm{SN}_{1}$ au $\mathrm{SN}_{2}$, en perdant pour une large part sa valeur sémantique fondamentale (Furukawa, 1996: 93); cf. aussi (Kupfermann, 2000: 32). Souvent d'ailleurs, coP peut fonctionner seul comme construction absolue (Riegel et al., $1998: 192)$ :

[23c] Il a la tête basse/ les cheveux noirs --> Pierre se promène la tête basse/ Blême, les cheveux noirs, elle s'appuyait sur le bras de mon frère.

Bref, du point de vue de l'apport sémantique de la co-prédication - et ces observations corroborent effectivement celles énoncées dans le point précédent, les divers tours se répartissent de la façon suivante sur l'axe allant de [- EXPANSION] vers [+ EXPANSION] : des constructions [T1, T2a] où le verbe de préd1 véhicule l'information centrale, nous passons à d'autres tours [T2b-c, T3a-b] où le verbe principal est un verbe recteur faible qui a besoin de l'appoint de coP. Enfin, dans le dernier groupe de coP-Adj [T4 et T5], préd1 se limite à jouer un rôle fonctionnel dans l'énoncé et c'est la co-prédication qui véhicule l'essentiel de l'apport sémantique.

\subsubsection{Interférences rectionnelles entre prédicats}

61 Ce paramètre permet d'évaluer le degré d'intégration de coP. Selon le degré d'interférence, nous distinguerons trois cas de figure :

\section{(a) absence d'interférence :}

62 Les adjectifs dans les tours T1 et T2a représentent des prédicats "accidentels ", des 'adjoints' non indispensables, contrairement à l'attribut du sujet qui est obligatoire après le verbe copule (Jean est furieux/ *Jean est) et en opposition avec les autres types de coPAdj [24c-c'] :

[24a] Le client est parti, furieux.

[24a'] Le client est parti.

[24b] Je bois le café chaud.

[24b'] Je bois le café.

[24c] Paul trouve la mesure indispensable.

[24c'] *Paul trouve la mesure. 
La proximité des tours T1 et T2a est soulignée par une similarité de fonctionnement dans des occurrences où le co-prédicat semble perdre son caractère " accidentel ». Ceci est illustré par les exemples [25a] et [25b] :

[25a] Hier soir, Jean est rentré ivre.

[25b] Son café, il le boit chaud.

Dans l'énoncé [25a], dérivé du tour T1, on remarquera que ivre est sous la portée de la négation de préd1, alors que déçu et blessé ne le sont pas [26a-b]. Il s'agirait donc plutôt d'un prédicat complexe (rentrer ivre) que de la connexion de deux prédicats « autonomes » (il rentre et il est ivre) cf. à ce propos aussi (Forsgren, 1993 : 19) et (Noailly, 1999: 127). L'argumentation est grosso modo similaire dans le cas de chaud lorsqu'il est sous la portée du prédicat [25b]. Il boit son café chaud n'implique pas seulement que le café est chaud quand il le boit, mais qu'il le boit chaud quand il en boit. Dans ce type d'énoncés, l'ensemble de la phrase matrice et de la prédication apposée doit être interprétable comme pratiquement une seule action langagière, un seul contenu informationnel pertinent. Muller (2000 : 31) relève d'ailleurs pour les tours T2a certains emplois quasi adverbiaux, tels que Il attache son chien serré, où l'emploi de l'adjectif s'approche de celui des "adjectifs employés adverbialement " (bronzer idiot, rouler japonais). Tout comme [25a], ces énoncés de type T2a acceptent une polarité négative spécifique et entrent sous la portée de la négation de préd1 $[26 \mathrm{c}-\mathrm{d}]$ :

[26a] Déçu et blessé, Jean n'est pas rentré ivre.

[26b] Déçu et blessé, Jean est rentré, mais pas ivre.

[26c] Son café, il ne le boit pas chaud.

[26d] Son café, il le boit, mais pas chaud.

Dans les deux tours T1, T2A, le contexte et la position des éléments paraissent jouer un rôle capital pour sélectionner les lectures « accidentelle » et « substantielle » :

[27a] Jeune, il mourra de mort violente.

[27b] Il mourra jeune de mort violente.

[27c] Il boit son café chaud.

[27d] Chaude, il ne la boira pas, cette tisane.

[27e] Il boit son café, noir comme une nuit sans lune.

[27f] En cas de rhume, il faut boire chaud.

CoP-Adj ne semble constituer un apport à préd1 que s'il se trouve après le prédicat verbal [27a-c] et qu'aucun autre élément ne vienne accentuer l'autonomie du co-prédicat [27e]. Bref, d'une façon générale, l'apport de l'adjectif prédicatif est accessoire par rapport au verbe recteur, mais sa position après le verbe peut générer un impact pragmatique de ce dernier sur le premier. L'interprétation 'intégrée' est donc soumise à de nombreuses conditions : ainsi, l'implication est inexistante quand l'adjectif prédicatif se trouve en tête de phrase, l'interprétation du sens de coP est alors plus facilement circonstancielle; de même, la nature sémantique de l'adjectif prédicatif ou la présence d'éléments accentuant ou neutralisant son autonomie prédicative [cf. 27e-f] influeront sur cet impact pragmatique. 


\section{(b) interférence limitée :}

[28c] Sylvie a les yeux bleus/*les yeux/*des yeux bleus.

[28d] Il y a une place de libre/ ?une place/.

\section{(c) interférence forte :}

Dans les constructions $\mathrm{T} 2 \mathrm{c}$ et $\mathrm{T} 3 \mathrm{a}$, les verbes régissants intègrent le coP adjectivant dans leur sémantisme. Ils expriment dès lors une valeur compositionnelle : le coP adjectivant change non seulement la structure argumentative du verbe principal mais s'intègre aussi dans son sémantisme. Ces verbes deviennent des causatifs-performatifs :

[29a] Le prof a rendu Jean nerveux ( $<=>$ le prof lui a rendu la feuille).

(= Il a fait que Jean est devenu nerveux)

[29b] Il qualifie la nouvelle de prématurée ( $<=>$ Ce diplôme ne vous qualifie pas pour assumer un tel poste).

(= Il fait par un acte de qualification que la nouvelle est considérée comme prématurée)

La gradation du pôle [- INTÉGRATION] vers le pôle [+ INTÉGRATION] se traduit ici par le degré d'attraction de l'adjectif prédicatif par le verbe régissant : dans T1 et T2a, l'interférence des prédications est accessoire, même si un impact pragmatique n'est pas exclu ; dans $\mathrm{T} 2 \mathrm{~b}, \mathrm{~T} 3 \mathrm{~b}, \mathrm{~T} 4$ et T5, la co-prédication comme entité globale est au contraire attirée dans la rection de préd1; dans $\mathrm{T} 2 \mathrm{c}$ et $\mathrm{T} 3 \mathrm{a}$, enfin, elle fournit un apport sémantique à la composition du prédicat principal même.

\subsection{Conclusions}

71 Le tableau ci-dessous résume le poids au sein des coP-Adj des contraintes fonctionnelles et rectionnelles déterminant le niveau d'intégration de préd2 dans préd1.

\begin{tabular}{|l|c|c|c|c|c|c|c|c|}
\hline & T1 & T2a & T2b & T2c & T3a & T3b & T4 & T5 \\
\hline Dépendance fonctionnelle & - & $-/+$ & + & ++ & ++ & + & + & $+/-$ \\
\hline
\end{tabular}




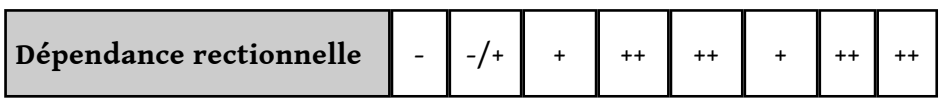

Tableau 2 : cohésion interprédicative

72 Cette grille nous montre que les constructions adjointes [T1] subissent le moins de contraintes, et peuvent être vues comme les moins intégrées du point de vue de la hiérarchisation entre prédications et de l'attraction de coP par le verbe régissant. Le tour avec des verbes occasionnellement attributifs [T2a] est peu contraint également, alors que les six autres tours expriment tous une dépendance fonctionnelle ou rectionnelle plus ou moins forte, mais de nature différente et sur divers plans.

73 Les verbes causatifs d'état [T2c] et les attributs indirects de l'objet introduits par une préposition [T3a] présentent l'attraction la plus forte de coP par préd1 et subissent, en conséquence, diverses contraintes fonctionnelles et rectionnelles fortes. Ces constructions nous paraissent, pour cette raison, présenter l'intégration la plus forte de coP. Les emplois attributifs avec avoir [T4], les verbes à attributs propositionnels [T2b] et les attributs indirects en comme |T3b] subissent en gros les mêmes contraintes mais elles sont moins rigoureuses. Les tours en de adj [T5] enfin présente parmi ce dernier type de constructions le plus de résistance à l'impact rectionnel de préd1 sur la prédication seconde (cf. sa résistance à la portée de la négation (3.1.1)).

\section{Synthèse}

74 La combinaison de deux échelles [+/- EXPANSIOn] et [+/- InTÉGRATION] permet de positionner les divers types de co-prédication adjectivantes par rapport à deux dimensions caractéristiques de la prédication seconde, le plan intraprédicatif (la cohésion entre les composantes de coP) et le plan interprédicatif (la cohésion entre les prédications première et secondaire).

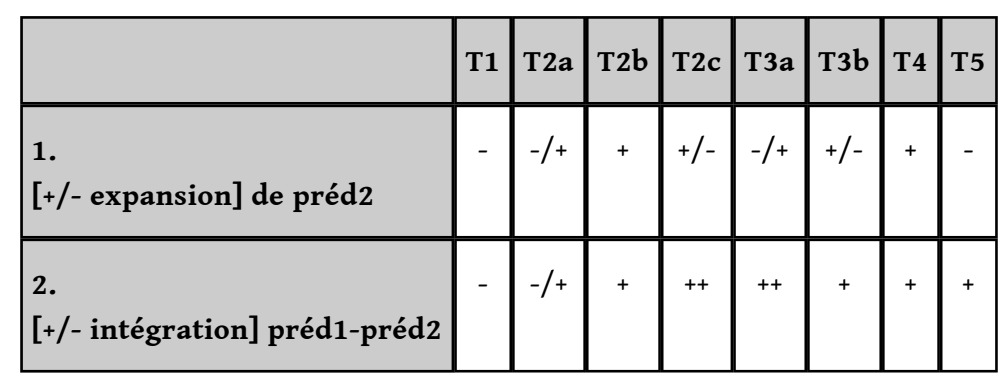

Tableau 3 : combinaison des plans intra et interprédicatif

Ces données combinées mettent en évidence trois orientations pour les tours considérés :

- les coP qui sont marquées des traits [- INTÉGRATION] et [- EXPANSION] sont celles qui sont traditionnellement le plus souvent associées à la fonction d'apposé ;

- les tours qui indiquent un degré élevé d'intégration et d'expansion correspondent plutôt à la fonction d'attribut de l'objet ou sont souvent analysés comme des clausules (small clauses) ;

- enfin, les coP qui combinent les traits [+ INTÉGRATION] et [- EXPANSION] sont celles dont le rattachement à la co-prédication est souvent le plus contesté (surtout T5). 


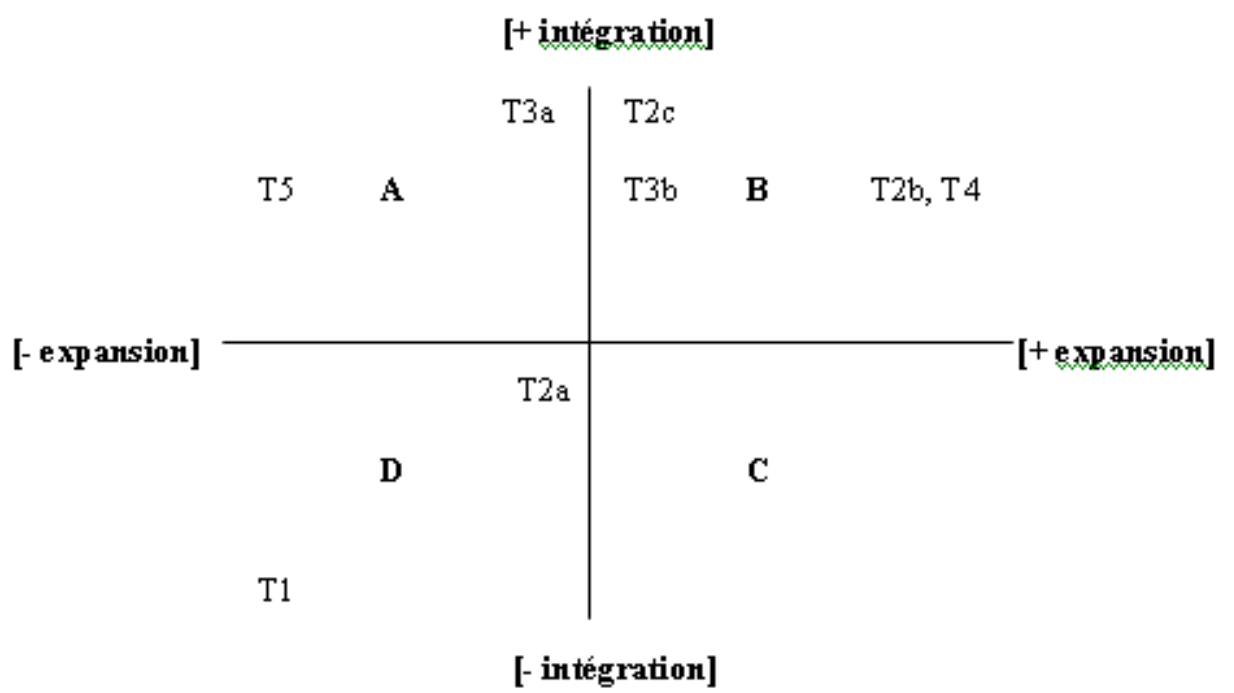

Figure 1 : localisation des types par rapport aux deux échelles

La localisation des coP-Adj dans les différents quadrants confirme les caractéristiques mises en évidence :

7 Le positionnement dans les quadrants $A$ et $B$ révèle la grande variété des tours marqués [+ intéGRATION]. Ceux-ci peuvent impliquer un haut degré d'expansion (les coP propositionnels de T2b ; les emplois attributifs avec avoir dans T4), un niveau moyen (les coP avec des verbes causatifs d'état dans T2c, les coP indirects en comme dans T3b) et même une tendance vers la réduction (verbes occasionnellement attributifs dans T3a, les tours en de adj dans T5). L'intégration semble donc être un trait particulièrement saillant de la majeure partie des coP-Adj ;

78 La localisation dans le quadrant $\mathrm{D}$ regroupe de son côté les tours qui affichent une orientation plutôt négative par rapport aux deux paramètres : les adjoints [T1] et les coP avec des verbes occasionnellement attributifs [T2a]. Les constructions qui présentent une cohésion limitée entre les prédications sont aussi celles qui proposent une cohésion peu contraignante aux composantes de coP ;

adrants $\mathrm{B}$ et $\mathrm{D}$ regroupent, comme nous l'avons déjà souligné, les deux grands types de fonctions associées à la coP : l'apposition et l'attribut de l'objet. Dans le quadrant $\mathrm{A}$, on retrouve les tours qui sont souvent situés à la frontière des arguments indirects de préd1, voire interprétés comme des compléments circonstanciels [30a] :

[30a] Paul m'a séduit par ses bonnes manières. (Cadiot 2000 : 112)

L'absence de coP-Adj dans le quadrant $\mathrm{C}$ indique une «lacune » dans le système des coPAdj., mais n'implique pas que d'autres tours ne pourraient y être localisés. La combinaison d'une cohésion interne forte et d'une intégration faible pourrait correspondre, par exemple, aux caractéristiques des constructions absolues, des coprédications comportant un thème et un prédicat autonomes [30b-c] :

[30b] Les petits passaient, la démarche vive. (Larbaud; Grevisse - Goosse, 1991 : §308 : .512). 
[30c] Les conséquences politiques d'un tel scrutin restant difficiles à évaluer, la plupart des partis ont laissé à leurs électeurs toute liberté de conscience. (Le Monde 12-13.06.2005 : 6).

81 En conclusion, les caractéristiques relevées indiquent que l'exploitation des paramètres de gradation de la dépendance et le traitement parallèle de la connexion propositionnelle et de la co-prédication donnent des résultats stimulants pour les types de coP-Adj. L'approche proposée pose par la même occasion un cadre partagé pour approfondir la distinction entre les deux domaines dans d'autres types de constructions et pour interpréter leurs particularités dans le fonctionnement discursif. Ainsi, par exemple, à partir des propriétés dégagées, nous pouvons formuler l'hypothèse que le trait [INTÉGRATION], combiné en particulier avec le trait [- EXPANSION], tend à favoriser un fonctionnement transphrastique de coP :

[31a] En Afghanistan, la révolte contre les mollahs se développait parmi les femmes. Défiant le pouvoir des talibans, deux jeunes femmes étaient parties faire des études aux USA.

[31b] C'est son père, René, qui avait pris en main et développé la station en profitant du boom des années 70. Décédé subitement d'un accident de la route en 1986, on craignait le pire pour la suite. (Béguelin, $2000: 323$ )

[31c] L'Afghanistan s'enfonçait lentement dans l'arriération et le sousdéveloppement. Défiant le pouvoir des talibans, deux jeunes femmes étaient parties faire des études aux USA.

De fait, des études sur les constructions participiales indiquent que les coP participiaux en position initiale, qui se situent donc dans l'intervalle entre une prédication qui vient d'être clôturée et une nouvelle prédication que la co-prédication introduit, peuvent marquer soit la continuité avec l'énoncé précédent $[31 \mathrm{a}, \mathrm{b}]$, soit la rupture informationnelle [31c] (Béguelin, 2000 : 323 ; Combettes, 1998 : 62).

\section{BIBLIOGRAPHIE}

BÉGUELIN, M.-J. 2000. De la phrase aux énoncés : grammaire scolaire et descriptions linguistiques.

Bruxelles : De Boeck Duculot.

BERRENDONNER, A., REICHLER-BÉGUELIN, M.-J. 1989. Décalages : Les niveaux de l'analyse linguistique. Langue française $81: 99-125$.

BLANCHE-BENVENISTE,C., CADDÉO, S. 2000. Préliminaires à une étude de l'apposition dans la langue parlée. Langue française $125: 60-70$.

CADIOT, P., FURUKAWA, N. 2000. Présentation. Langue française 127 :3-5.

CADIOT, P. 2000. La préposition comme connecteur et la prédication seconde. Langue française 127 : 112-125.

COMBETTES, B. 1998. Les constructions détachées en français. Paris : Ophrys. 
FORSGREN, M. 1993. L'adjectif et la fonction d'apposition : observations syntaxiques, sémantiques et pragmatiques. L'Information grammaticale $58: 15-22$.

FORSGREN, M. 2000. Apposition, attribut, épithète : même combat prédicatif ? Langue française 125 : $30-45$.

FURUKAWA, N. 1996. Grammaire de la prédication seconde. Louvain-la-Neuve : Duculot.

GREVISSE, M., GOOSSE, A. 1991. Le bon usage. Paris - Louvain-la-Neuve : Duculot. (édition refondue par André Goosse).

GUIMIER, E. 1998.Les constructions à prédicat de l'objet des verbes de perception. Prédication, assertion, information, M. Forsgren, K. Jonasson, H. Kronning (éds). Uppsala : Acta Universitatis Upsaliensis. 231-241.

HAVU, E. 2002. L'interprétation des constructions détachées. Cercle de linguistique appliquée à la communication 10, /no10/havu.htm.

HAVU, E., PIERRARD, M. 2004. La prédication seconde en français : définition et extension du domaine. Linguistics Today - Facing a Greater Challenge (incl. CD Rom), P. van Sterkenburg (éd.). Amsterdam /Philadelphia : John Benjamins Publishing Company CD-Rom : contributions \S7_EvaHavuAndMichelPierrard_LaPredicationSecondeEnFrancais.pdf HAVU, E., PIERRARD, M. (à paraître). Prédications secondes adjectivales et participiales : l'interprétation des participes présents adjoints. Actes du colloque Représentation du sens III, Bruxelles, 3-5 novembre 2005.

KUPFERMAN, L. 2000. Avoir et prédication seconde. Langue française 127 : 67-85.

LAGAE, V. 1998. Les constructions en 'de+adjectif. Typologie et analyse. Louvain : Presses Universitaires de Louvain.

LEHMANN, C. 1989. Towards a typology of clause linkage. Clause combining in grammar and discourse, J. Haiman, S.A. Thompson (éds). Amsterdam/ Philadelphia : J. Benjamins. 181-225.

MULLER, C. 2000. Les constructions à adjectif attribut de l'objet, entre prédication seconde et complémentation verbale. Langue française $127: 21-35$.

NOAILLY, M. 1999. L'adjectif en français. Paris : Ophrys.

PIERRARD, M. 2002. Attribut de l'objet et verbe vecteur d'attribution : la place des constructions à valeur causative. Le système des parties du discours. Sémantique et syntaxe, R. Lowe (éd.). Laval : Les Presses de l'Université Laval. 358-369.

RAIBLE, W. 2001. Linking clauses. Language typology and language universals : an international handbook. Sprachtypologie und sprachliche Universalien : ein internationales Handbuch. La typologie des langues et les universaux linguistiques : manuel international, M. Haspelmath, K. Ekkard, W. Oesterreicher (éds.). Berlin : W. De Gruyter. 590-617.

RIEGEL, M. 1991. Pour ou contre la notion grammaticale d'attribut de l'objet : critères et arguments. À la recherche de l'attribut, M.-M. De Gaulmyn, S. Rémi-Giraud (éds). Lyon : Presses Universitaires de Lyon. 99-118.

RIEGEL, M. et al. 1998 (1 ${ }^{\text {re }}$ éd. 1994). Grammaire méthodique du français. Paris : Presses Universitaires de France.

WILMET, M. 2003. (1 ${ }^{\text {re }}$ éd. 1997) Grammaire critique du français. Bruxelles : Duculot. 


\section{NOTES}

1. Sous le terme adjectivant, nous regroupons non seulement les formes strictement adjectivales mais aussi des participes présent ou passé.

\section{RÉSUMÉS}

L'objectif de cette contribution est de proposer un cadre permettant d'appréhender la multiplicité des paramètres qui gouvernent les importantes différences d'extension et de flexibilité des tours regroupés sous l'étiquette des co-prédications (coP) adjectivantes: [1] les adjoints, [2] les attributs directs de l'objet avec des verbes occasionnellement attributifs, des verbes à attributs propositionnels et des verbes causatifs d'état, [3] les attributs indirects de l'objet introduits par une préposition ou par comme, [4] les emplois attributifs avec avoir et [5] les tours en de adj. L'approche suggérée exploite deux types de paramètres avancés pour décrire les différents degrés de connexion entre propositions (Lehmann, 1989). Le degré de réduction de la co-prédication permettra tout d'abord de déterminer la cohésion intraprédicative de celle-ci dans les différents tours. Le degré d'intégration de la co-prédication dans la prédication principale précisera pour sa part la cohésion interprédicative des divers énoncés produits.

This paper proposes a basis for the description of the numerous parameters governing the extension and flexibility of constructions classified as adjectival co-predications: [1] adjuncts, [2] direct object complements introduced by occasionally predicative verbs, by verbs taking propositional complements and by stative causative verbs, [3] indirect object complements introduced by a preposition or by comme, [4] the predicative uses of avoir and [5] de adj constructions. The approach is based on two types of parameters describing the different degrees of connection between propositions (cf. Lehmann [1989]): a) the degree of reduction of the copredication enables the definition of its intrapredicative cohesion in different constructions; $b$ ) the degree of integration of the co-predication in the main predication determines the interpredicative cohesion between the predications.

\section{INDEX}

Mots-clés : apposition, attribut de l'objet, co-prédication adjectivante, épithète détachée, prédication seconde

Keywords : adjectival co-predication, apposition, detached attributive adjective, object complement, secondary predication

\section{AUTEURS}

\section{EVA HAVU}

Université de Helsinki/Université Paris 3-CIEH, 1 rue Censier, 75005 Paris eva.havu@helsinki.fi 


\section{MICHEL PIERRARD}

Vrije Universiteit Brussel, Taal- en Letterkunde, Pleinlaan 2, B-1050 Brussel michel.pierrard@vub.ac.be 